\title{
Números cromossômicos em Kleberiella V.P. Castro \& Cath. (Orchidaceae, Oncidiinae) e gêneros afins
}

Túlio Luiz Laitano Penha ${ }^{1,2}$, Andréa Macêdo Corrêa ${ }^{1}$ e Eduardo Luís Martins Catharino ${ }^{1}$

Recebido em 16/12/2010. Aceito em 16/05/2011

\section{RESUMO}

(Números cromossômicos em Kleberiella V.P. Castro \& Cath. (Orchidaceae, Oncidiinae) e gêneros afins). Este trabalho teve como objetivo principal realizar a análise citotaxonômica de espécies filogeneticamente relacionadas dentro dos gêneros Kleberiella, Alatiglossum e Neoruschia com base na determinação dos seus números cromossômicos a partir dos seus meristemas radiculares. Foram obtidos os números cromossômicos de todas as espécies de Kleberiella (seis espécies), Neoruschia (monoespecífico) e de quatro das nove espécies conhecidas para Alatiglossum stricto sensu. Exceto para K. longipes, A. barbatum e A. micropogon, os quais corroboram com resultados anteriores, todos os outros números cromossômicos são inéditos. A contagem cromossômica se mostrou como um caráter taxonômico útil na delimitação de Alatiglossum fuscopetalum $(2 n=52)$ e do gênero Neoruschia $(2 n=48)$ das outras espécies analisadas. Entretanto não se mostrou eficiente para delimitação entre as espécies de Kleberiella (todas com $2 n=56$ ) e para separar este gênero de Alatiglossum no qual a maioria das espécies analisadas apresentou $2 n=56$. O numero básico sugerido para Alatiglossum e Kleberiella é $\mathrm{x}=28$ e para Neoruschia $x=24$.

Palavras-chave: Oncidium, Gomesa, citotaxonomia, números básicos

\begin{abstract}
(Chromosome numbers in the genus Kleberiella VP Castro \& Cath. (Orchidaceae, Oncidiinae) and related genera). This work aimed to provide cytotaxonomical analysis of species phylogenetically related within the genera Kleberiella, Alatiglossum and Neoruschia based on the determination of chromosome numbers from root meristems. We obtained the chromosome numbers of all species of Kleberiella (six species), Neoruschia (monospecific) and four of the nine known species of Alatiglossum stricto sensu. Except for K. longipes, A. barbatum and A. micropogon, which corroborate previous results, all other chromosome numbers are unpublished. Chromosome counting proved useful as a taxonomic character in the delimitation of Alatiglossum fuscopetalum $(2 n=52)$ and the genus Neoruschia $(2 n=$ 48 ) from the other species analyzed. However it was not efficient for delimitation between Kleberiella species (all with $2 n=56$ ) and to separate this genus from Alatiglossum in which most of the species studied had $2 n=56$. The provable basic number for Alatiglossum and Kleberiella is $x=28$ and for Neoruschia $x=24$.
\end{abstract}

Key words: Oncidium, Gomesa, cytotaxonomy, basic number

\section{Introdução}

A subtribo Oncidiinae (Orchidaceae, Epidendroideae, Cymbidieae) é a segunda maior subtribo de orquídeas das Américas com aproximadamente 1.700 espécies de distribuição exclusivamente neotropical. Possui uma destacada importância econômica com um alto número de espécies ornamentais, principalmente nos gêneros Miltonia, Odontoglossum e Oncidium Sw. os quais, juntos, compreendem mais da metade das espécies da subtribo Oncidiinae (Chase et al. 2003, 2005).

As espécies de Oncidiinae podem ser caracterizadas morfologicamente por apresentarem prefoliação condupli- cada, inflorescência axial basal e polinário completo com duas (Dressler 1993) a quatro polínias cartilaginosas, com inclusão de Ornitocephalinae. A maioria de suas espécies é epífita, enquanto outras são terrícolas ou litofíticas. Possuem diversos padrões de polinização como pseudo-cópula, produção de néctar ou óleos florais, mas a mimetização de flores que produzem alimento e/ou óleos florais tem sido relatada como a estratégia mais comum (Chase et al. 2009).

Tradicionalmente os limites genéricos em Oncidiinae foram baseados nos tratos florais (Williams et al. 2001). Por outro lado, atualmente tem sido ressaltado que os caracteres florais não devem ser utilizados isoladamente para definir limites genéricos dentro da subtribo e que, ao invés destes,

\footnotetext{
1 Instituto de Botânica de São Paulo, Núcleo do Orquidário Frederico Carlos Hoehne, São Paulo, SP, Brasil

2 Autor para correspondência: tulio_laitano@yahoo.com.br
} 
os caracteres vegetativos e os números cromossômicos deveriam ser enfatizados na delimitação de gêneros em Oncidiinae (Chase et al. 2009).

Entretanto, mesmo que Oncidiinae seja uma das subtribos de Cymbidieae com maior número de informações citológicas da família (Chase et al. 2005), existem poucos estudos aprofundados sobre o cariótipo com enfoque na caracterização de gêneros e espécies, como os realizados por Chase \& Palmer (1992), Dematteis (1997) e Félix \& Guerra (2000). Os trabalhos realizados demonstram que há uma inconsistência entre os caracteres florais, caracteres vegetativos e números cromossômicos. Gêneros formados tradicionalmente por características florais são polimorfos em relação aos caracteres vegetativos e números cromossômicos, e espécies vegetativamente similares, com os mesmos números cromossômicos, são colocadas em gêneros separados pela análise de suas flores (Chase 1986; Williams et al. 2001).

Recentemente, estudos filogenéticos têm demonstrando que muitos gêneros de Oncidinae em sua circunscrição tradicional como Erycina Lindl., Miltonia Lindl., Odontoglossum Kunth, Oncidium Sw., Tolumnia Raf., Trichocentrum Poepp. \& Endl. e Gomesa R.Br. são polifiléticos ou parafiléticos. Estes gêneros apresentam espécies com relação filogenética mais próxima de outros gêneros do que com a espécies tipo do gênero que se encontra (Williams et al. 2001). Por exemplo, todas as seções "brasileiras" de Oncidium Sw. como Waluewa, Crispa, Barbata, Bicoloria, Ranifera e Rhinocerus são mais próximas filogeneticamente de Gomesa do que da espécie tipo de Oncidium (Chase et al. 2009).

Muitas propostas de circunscrição de gêneros foram realizadas recentemente em Oncidiinae, para rearranjar as espécies que não tinham relações filogenéticas dentro do gênero que se encontrava. Em relação às seções brasileiras de Oncidium e do gênero Gomesa duas escolas divergentes atuam. Uma escola, denominada de "lumper" propõe com base principalmente em dados moleculares a inclusão das seções brasileiras citadas de Oncidium e outros gêneros relacionados como Baptistonia Barb Rodr., Binotia Rolfe, Ornithophora Barb. Rodr., Rodrigueziella Kuntze e Rodrigueziopsis Schltr. sob Gomesa, por formarem um clado monofilético denominado "Gomesa" que, nesse senso amplo, possuiria cerca de 125 espécies (Chase et al. 2009; Pridgeon et al. 2009; Penha \& Catharino 2010).

Já a outra escola, denominada "spliter", propõe reorganizar estas seções restabelecendo gêneros mais antigos como Baptistonia Barb Rodr. e Coppensia Dumort e descrevendo novos gêneros baseados em caracteres morfológicos e nos estudos filogenéticos publicados, como Alatiglossum Baptista, Brasilidium Campacci, Carenidium Baptista e Rhinocidium Baptista, (Catharino \& Campacci 2009) os quais foram segregados em trabalhos posteriores e ainda existe discussão de como reorganizar estas espécies. Por exemplo, Alatiglossum Baptista foi inicialmente estabelecido com 18 espécies, antes reconhecidas na seção Barbata Lindl. ex Pfitz
(Oncidium l.s.) (Docha Neto et al. 2006). Posteriormente, foram propostos mais dois gêneros baseados em caracteres vegetativos para agrupar dois complexos de espécies em Alatiglossum: Kleberiella Castro \& Cath., com seis espécies e Neoruschia Cath. \& Castro, monoespecífico (Castro Neto $\&$ Catharino 2006).

As espécies dos gêneros Alatiglossum stricto sensu e Kleberiella em uma primeira análise filogenética, utilizando-se os segmentos de DNA ITS 1 e 2 (Fig. 1), foram reconhecidas dentro de um mesmo clado denominado "Barbata" (Williams et al. 2001; Faria 2004). Em um nova análise, incorporando outras regiões do DNA (Fig. 2), Neoruschia e Carenidium gracile foram incluídos no clado "Barbata" (Chase et al. 2009). Porém, C. gracile foi considerado dentro do clado "Concoloria" na primeira análise, com outra espécie de Carenidium, C. concolor, com a qual possuí características morfológicas semelhantes como lobos laterais não desenvolvidos, calos no labelo consistindo em duas lamelas, ausência de tabula infraestigmática e polínias com mais de $0,3 \mathrm{~mm}$ de comprimento (Faria 2004). Por não apresentar características em comum com as espécies do clado "Barbata" um novo gênero foi descrito para C. gracile, Nitidocidium (Barros \& Rodrigues 2010). Entretanto, apesar das diferenças morfológicas neste mesmo trabalho foi proposta a inclusão de Neoruschia em Alatiglossum.

Por outro lado, a expansão do conceito de Gomesa necessitaria de divisões subgenéricas, para caracterização de grupos morfológicos, as quais não foram propostas em cima dos clados internos bem suportados (Chase et al. 2009). A caracterização de grupos morfológicos de espécies filogeneticamente relacionadas facilita estudos entre a delimitação de táxons (Penha 2011). Assim, como não foram realizadas divisões subgenéricas em Gomesa lato sensu a adoção de uma circunscrição menor com espécies filogeneticamente relacionadas é mais propícia para estudar a variação do número cromossômico no grupo, até que sejam propostas divisões infragenéricas em Gomesa lato sensu.

Além das divergências sobre a circunscrição genérica, também existem problemas de delimitação em nível hierárquico de espécie, como no complexo de espécies que vieram a formar Kleberiella. Destaca-se que, morfologicamente, as espécies que constituem o gênero Kleberiella são muito confundidas entre si, pois todas apresentam pseudobulbos elípticos alongados com uma ou duas folhas no ápice, uma a duas bainhas foliáceas na base, inflorescência racemosa formada antes da formação completa dos pseudobulbos, com flores muito semelhantes entre si (Penha 2011). Após uma comparação morfológica, Teuscher (1968) ressaltou que o número cromossômico poderia suportar a integridade de $K$. longipes como uma entidade distinta de $K$. uniflora. Entretanto, uma única espécie do gênero, $K$. longipes, tem o número diplóide conhecido, $2 n=56$ (Félix \& Guerra 2000).

Em muitos casos, a simples determinação do número cromossômico fornece informações importantes para a compreensão da filogenia, evolução (Stebbins 1971) e ca- 


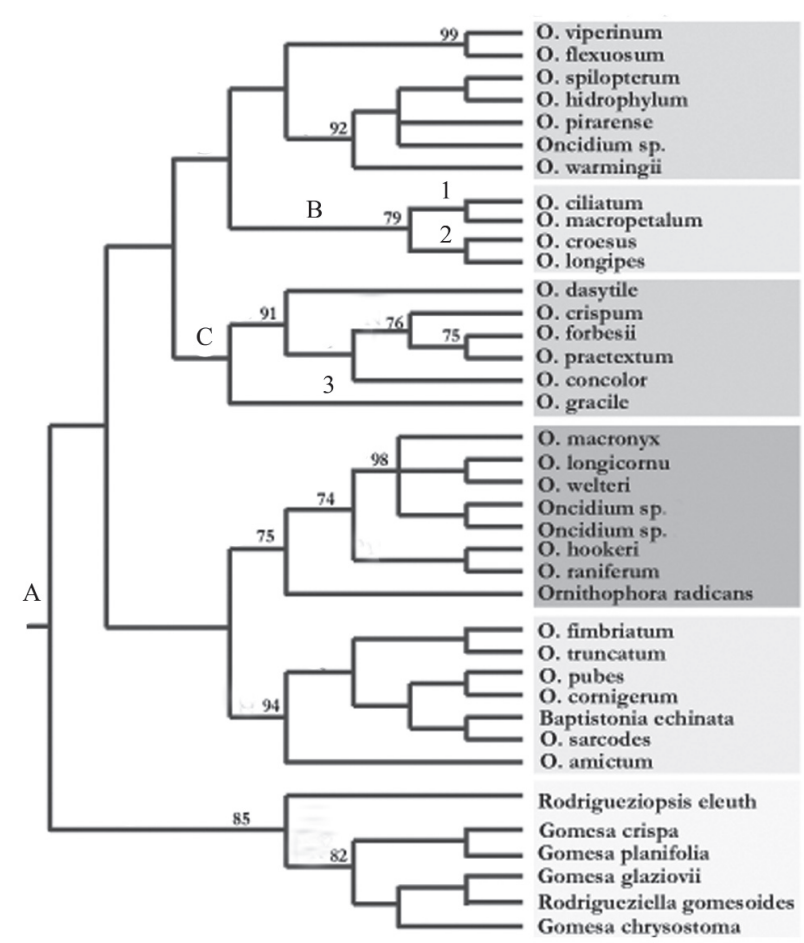

Figura 1. Árvore filogenética de consenso estrito do clado "Gomesa" gerado a partir de dados morfo-anatômicos, químicos e macromoleculares (ITS 1 e 2). (A) Clado "Gomesa". (B) Clado "Barbata". (C) Clado "Concoloria". (1) Alatiglossum Baptista stricto sensu. (2) Kleberiella V.P.Castro Neto \& Cath.. (3) Carenidium gracile (Lindl.) Baptista. Fonte: Faria (2004).

racterização de gêneros e espécies de difícil delimitação em Orchidaceae, como em outras famílias de angiospermas, desde que associada a outras abordagens como filogenia, morfologia e distribuição geográfica (Stace 1989; Corrêa 2003, Cozzollino et al 2004; Francesca \& Aquaro 2008; Koehler et al. 2008; Chase et al. 2009; Soltis \& Soltis 2009). Assim, o objetivo deste trabalho foi verificar a variação do número cromossômico em espécies de Kleberiella e de algumas espécies de gêneros relacionados filogeneticamente dentro do clado Barbata (Alatiglossum, Neoruschia e Carenidium), verificando se este número pode ser utilizado na taxonomia do grupo, corroborando ou não alguma das circunscrições genéricas propostas para o clado, bem como fornecer dados inéditos para compreensão da evolução cromossômica numérica em Oncidiinae

\section{Materiais e métodos}

Foram estudadas todas as espécies conhecidas de Kleberiella e de Neoruschia: K. croesus (Rchb.f.) V.P.Castro \& Cath., K. longipes (Lindl.) V.P.Castro \& Cath., K. regentii (V.P.Castro \& Carr) V.P.Castro \& Cath., K. bohnkiana (V.P.Castro \& Carr) V.P.Castro \& Cath., K. unicolor (Rolfe) V.P.Castro \& Cath., K. uniflora (Lindl.) V.P.Castro \& Cath., N. cogniauxiana (Schltr.) Cath. \& V.P.Castro, parte das espécies de Alatiglossum: A. barbatum (Lindl.) Baptista,
A. ciliatum (Lindl.) Baptista., A. fuscopetalum (Hoehne) Baptista, A. micropogon (Rchb. f.) Baptista e Carenidium gracile (Lindl.) Baptista.

Para a análise cromossômica foram utilizadas raízes frescas das plantas em cultivo no Orquidário Frederico Carlos Hoehne, Núcleo do Orquidário, Instituto de Botânica de São Paulo, já existentes ou coletadas através de expedições direcionadas. Todos os materiais utilizados foram relacionados (Tab. 1). Os materiais testemunhos dos indivíduos analisados foram depositados nos Herbários Maria Eneida P. Kauffman Fidalgo (SP) e D. Bento Pickel (SPSF).

Os meristemas radiculares foram pré-tratados com 8-Hidroxiquinoleína $0,002 \mathrm{M}$ e armazenados por 24 horas em geladeira a $5^{\circ} \mathrm{C}$ e depois fixados em Carnoy (ácido acético glacial : etanol, 1:3) por mais 24 horas. Posteriormente o material foi armazenado em freezer.

Para o preparo das lâminas, as raízes foram lavadas três vezes em água destilada, por 5 minutos, hidrolizadas por 30 minutos em HCL $5 \mathrm{~N}$ e, em seguida, lavadas em água destilada. Os meristemas foram esmagados em ácido acético (60\%) sob lamínula, que foi posteriormente retirada após imersão em nitrogênio líquido. As lâminas foram secas e coradas seguindo a técnica de Giemsa por 30 minutos e depois seladas com resina sintética Entellan, para a fixação da lamínula (Guerra 1983). As lâminas permanentes montadas foram examinadas em microscópio ótico e fotomicrografadas com câmera digital Canon EOS 10D para análise e apresentação dos resultados. Para a certificação dos resultados foram analisados no mínimo três indivíduos de cada espécie e contadas pelo menos 10 células por indivíduo analisado.

Para discussão dos resultados foi realizada uma revisão dos números cromossômicos em Oncidium l.s. e alguns gêneros afins publicados em: (Blumenschein 1960; Tanaka \& Kamemoto 1984; Moore 1973; 1974; 1977; Charanasri \& Kamemoto 1975; Charanasri et al. 1973; Sinotô 1969; Goldblatt 1984; 1985; Goldblatt \& Johnson 1988; 1990; 1991; 1994; 1996; Félix \& Guerra 2000; Davinã et al. 2009).

\section{Resultados e discussão}

\section{Variação do número cromossômico}

A variação dos números cromossômicos encontrada foi de $2 n=48,52$, 54 e 56 sendo Neoruschia $2 n=48$, Alatiglossum fuscopetalum $2 n=52$, Carenidium gracilii $2 n=54$ e $2 n=56$ para as demais espécies analisadas (Tab. 1, Fig. 3 e 4). Não foram encontradas variações entre os indivíduos analisados da mesma espécie. Exceto pelas espécies Alatiglossum barbatum, A. micropogon e Kleberiella longipes, para as quais nossos dados corroboram com a literatura, as demais contagens são inéditas. Foram observados satélites em K. croesus (Fig. 3A ) e K. bohnkiana (Fig. 3D).

Verifica-se que a contagem dos números cromossômicos pode ser empregada como um caráter citotaxonômico para 


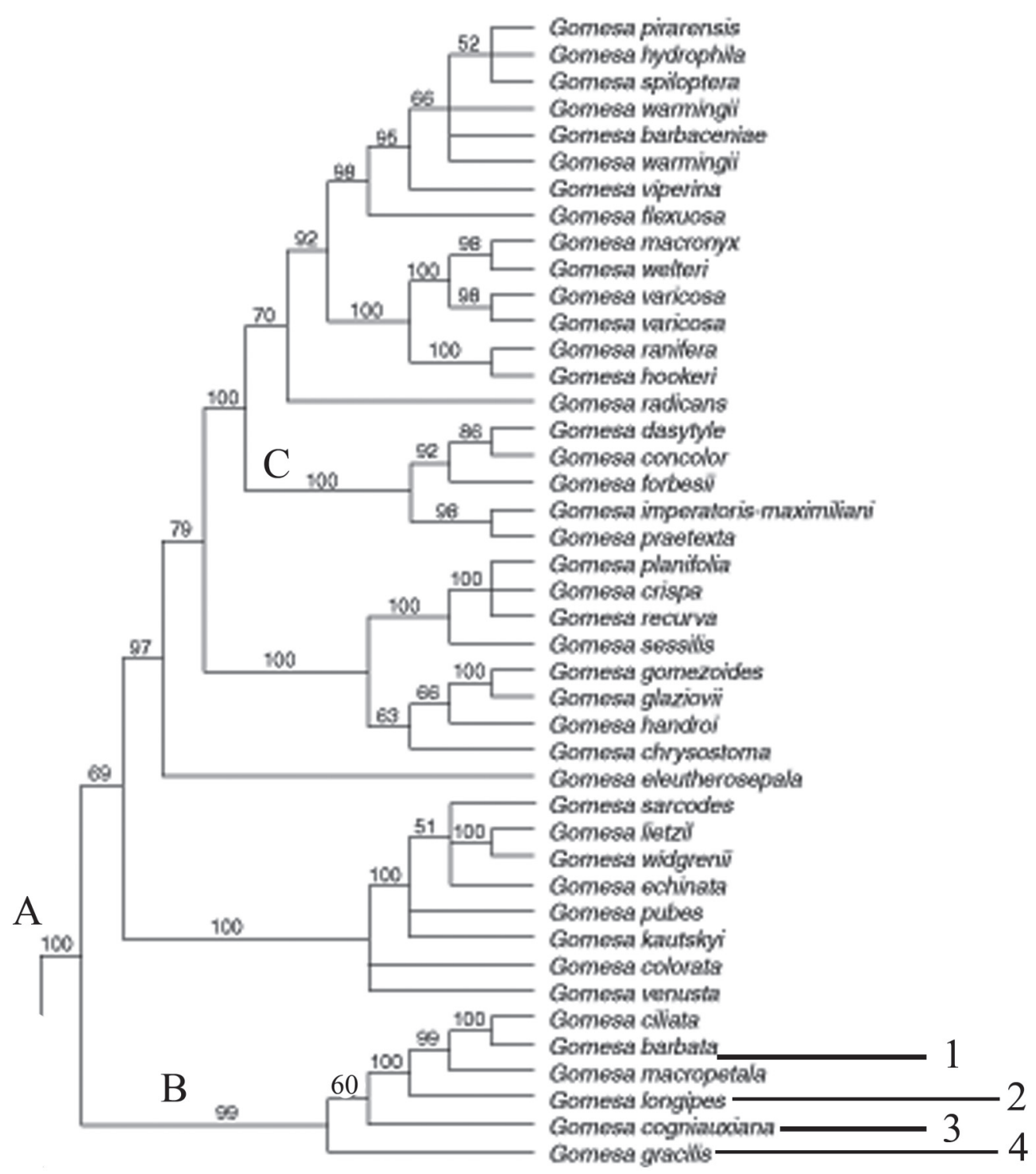

Figura 2. Árvore filogenética de consenso estrito do clado "Gomesa" gerada a partir de dados macromoleculares (plastídio, ITS 1 e 2). (A) Clado "Gomesa” (B) Clado "Barbata". (1) Alatiglossum stricto sensu. (2) Kleberiellla. (3) Carenidium gracile. Fonte: Chase et al. (2009).

diferenciar Alatiglossum fuscopetalum $2 n=52$, Neoruschia cogniauxiana $2 n=48$ e Carenidium gracile $2 n=54$, enquanto todas as outras espécies analisadas apresentaram $2 n=56$. Estes táxons são os que apresentam maior dissimilaridade morfológica entre as espécies estudadas, sendo que A. fuscopetalum é a única espécie que ocorre no cerrado, C. gracile é a única terrícola e que apresenta as sépalas laterais livres, ausência da tabula infraestigmática e calosidade do labelo formada apenas por duas quilhas, característica incomum para as outras espécies do clado. O gênero Neoruschia também apresenta as sépalas laterais livres, mas com calosidade diferenciada, formada por nove pequenas protuberâncias em formato cilíndrico. Diferentemente, as espécies de Alatiglossum stricto sensu e Kleberiella apresentam as sépalas laterais parcialmente conatas e calos em forma de crista (Castro Neto \& Catharino 2006).
Por outro lado, apenas a contagem cromossômica não foi suficiente para ser utilizada como um caráter na delimitação entre as espécies similares entre si, como as espécies de Kleberiella, as quais apresentaram $2 n=56$ e as espécies de Alatiglossum (A. barbatum, A. ciliatum e A. micropogon), também com $2 n=56$. Entretanto, dentro destes dois gêneros as espécies podem se cruzar entre si, mas não entre os gêneros (Penha 2011), sendo que algum outro fator, além do número cromossômico, deve ser responsável pela incompatibilidade entre os gêneros. A utilização de técnicas mais sofisticadas como o bandamento ou a hibridização in situ poderão revelar diferenças nas estruturas dos cromossomos entre as espécies morfologicamente similares e que apresentaram o mesmo número cromossômico. Estes métodos facilitam a visualização de diferenças na estrutura do cromossomo e foram empregados com sucesso na carac- 
Tabela 1. Espécies analisadas dos gêneros Alatiglossum Baptista, Carenidium Baptista, KleberiellaV.P.Castro\&Cath. e NeoruschiaCath. \&V.P.Castro, coleçõestestemunha procedentes do Brasil e seus correspondentes números cromossômicos (2n). BA: Bahia, GO: Goiás, MG: Minas Gerais, RJ: Rio de Janeiro, SP: São Paulo (s.p. = sem procedência)

\begin{tabular}{|c|c|c|}
\hline Táxon & Coleção-testemunha & $2 n$ \\
\hline A. barbatum(Lindl.) Baptista & BA, Noblicks.n. (SP 195613) & 56 \\
\hline A. ciliatum(Lindl.) Baptista & MG, Tabuleiro, Laitano 61 (SPSF) & 56 \\
\hline A. fuscopetalum(Hoehne) Baptista & GO, Goiânia, Laitano 141 (SPSF) & 52 \\
\hline A. micropogon(Rchb. f.) Baptista & RS, Porto Alegre, Catharino s.n. (SPSF) & 56 \\
\hline C. gracile(Lindl.) Baptista & MG, Barbacena Laitano 160 (SPSF) & 54 \\
\hline K. bonhkiana(V.P.Castro\&Carr)V.P.Castro\&Cath. & BA, São José da Vitoria, Bonhke s.n. (SP 382718) & 56 \\
\hline K. longipes(Lindl.) V.P.Castro\&Cath. & SP, Ubatuba, Laitano 91 (SPSF) & 56 \\
\hline K. regentii(V.P.Castro\&Carr)V.P.Castro\&Cath. & BA, Camacã, Martins s.n. (SP 376984) & 56 \\
\hline K. uniflora(Lindl.) V.P.Castro\&Cath. & MG, Serra do Cipó, Laitano 121 (SP) & 56 \\
\hline K. unicolor(Rolfe)V.P.Castro\&Cath. & RJ, Rio de Janeiro, Bicalho s.n. (SPSF 44290) & 56 \\
\hline N. cogniauxiana(Schltr.) Cath.\&V.P.Castro & SP, São José do Barreiro, Laitano 25 (SP) & 48 \\
\hline
\end{tabular}

terização de cariótipos entre espécies com o mesmo número cromossômico e revelaram detalhes sobre a evolução e a diversidade de espécies em Orchidaceae e outras famílias de plantas (Dematteis 1997; Corrêa 2003; Cozzollino et al. 2004; Koehler et al. 2008).

Os dados aqui apresentados levam a crer que Kleberiella pode ser considerado como pertencente ao gênero Alatiglossum, conforme foi publicado originalmente, pois ambos apresentam indivíduos com $2 n=56$ (Fig. 3 e 4) e $100 \%$ de sustentação pelas análises filogenéticas (Fig. 1 e 2). As espécies que formam os gêneros Kleberiella e Alatiglossum, tanto no aspecto vegetativo como no reprodutivo, são os mais similares dentro do clado "Barbata", porém podem ser distinguidos entre si pelas características vegetativas (Castro Neto \& Catharino 2006). Por outro lado, a inclusão de Kleberiella e Neoruschia em Alatiglossum, proposta por Barros \& Rodrigues (2010), não pode ser mantida sem Nitidocidium gracile, pois Alatiglossum se torna parafilético. Como observado (Fig. 2), sem N. gracile o clado apresenta apenas $60 \%$ de sustentação. Entretanto, com a sua inclusão passa para 99\% de sustentação. A posição de $N$. gracile no clado "Barbata" é questionável, uma vez que foi considerado em dois clados diferentes dentro do clado "Gomesa".

Se for mantida a relação filogenética de $N$. gracile no clado "Barbata", as opções que podem ser tomadas para a manutenção de gêneros bem sustentados seriam: (1) a adoção de três gêneros para o clado "Barbata": Nitidocidium, caracterizado por plantas terrestres, tabula infraestigmática ausente, sépalas laterais livres e $2 n=54$; Neoruschia, caracterizado pelas sépalas laterais livres, sem cílios no istmo, pseudobulbo de textura rugosa e $2 n=48$; e Alatiglossum, com pseudobulbos de textura lisa, sépalas conatas parcialmente, cílios no istmo do labelo e $2 n=56$; (2) inclusão de Nitidocidium e Neoruschia em Alatiglossum; ou (3) adoção de todos estes gêneros, assim como os outros relacionados dentro do clado "Gomesa", sob Gomesa.

Estas opções são baseadas em dados filogenéticos, morfológicos e no número cromossômico. A variação do número cromossômico encontrada no clado "Barbata" foi de $2 n=48,52,54$ e 56 com a maioria das espécies apresentando $2 n=56$, o número mais comum dentro dos clados internos do clado "Gomesa" e de Oncidiinae em geral (Félix \& Guerra 200). Observamos que se mantido o senso expandido de Gomesa a delimitação deste gênero será polimórfica em relação às características morfológicas e números cromossômicos.

Entretanto, a manutenção dos clados internos maiores também seria polimórfica, como pode ser observado no clado "Barbata" que para poder ser individualizado por características vegetativas e números cromossômicos teriam que ser excluídos do clado Neoruschia e Nitidocidium. Os outros clados internos, também podem ser reconhecidos por características morfológicas e pelos números cromossômicos como o clado "Baptistonia", que possui plantas com pseudobulbo fusiformes, tabula infraestigmática ausente, borda do clinândrio elevada (Faria 2004) e $2 n=56$. Para caracterizar melhor grupos morfológicos com números cromossômicos teriam que ser separados clados menores e, se estes fossem utilizados estabelecer limites genéricos, seria necessário descrever novos gêneros. Ressalte-se, entretanto, que ainda existe uma grande lacuna de dados sobre as espécies do clado "Gomesa" e novos estudos seriam necessários para uma melhor caracterização dos clados internos neste grupo.

\section{Evolução cromossômica numérica}

A variação do número cromossômico encontrada no clado "Barbata" foi semelhante a maioria dos outros clados de "Gomesa”, os quais apresentam variações de $2 n=52$ e 56 . Porém, no clado "Synsepala" (Fig. 2) foram observadas muitas espécies poliplóides (Tab.2). Outras exceções são em relação à Rhinocidium macronix com $2 n=42$, Neoruschia cogniauxiana $2 n=48$, Carenidium gracile $2 n=54$ e Baptistonia pubes $2 n=84$. A variação cromossômica total encontrada no clado "Gomesa" é de $2 n=42$, $48,52,54,56,84,112,140$ e 168. Esta variação parece ter como número base $x=28 \mathrm{com}$ as séries de poliplóide de $3 x=84,4 x$ $=112,5 x=140$ e $6 x=168$. Os números $2 n=42,48,52$ e 54 podem ser resultado de perda ou fusão de pares cromossômicos. 

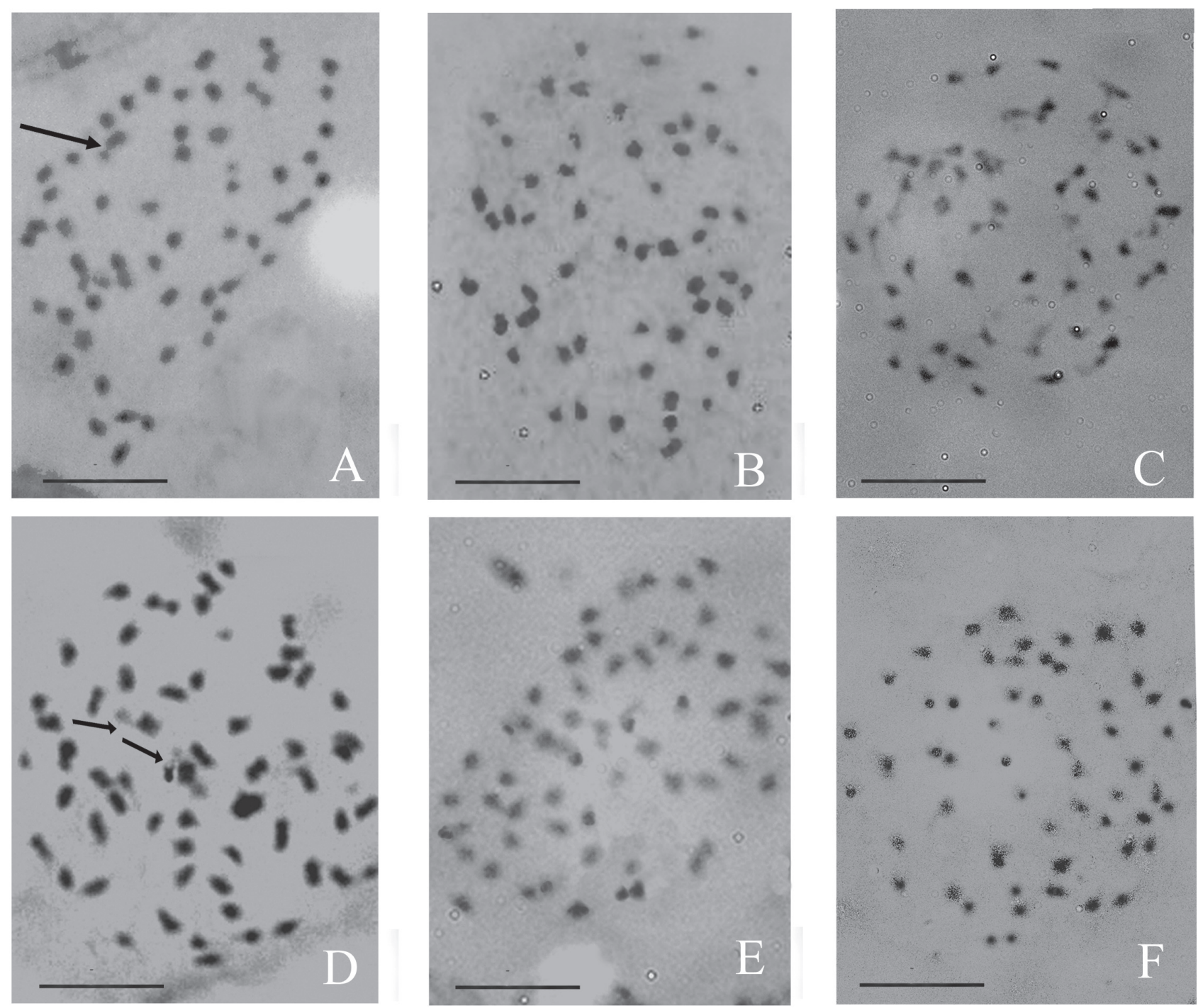

Figura 3. Fotos dos cromossomos das espécies: (A) Kleberiella regentii (V.P.Castro \& G.F.Carr) V.P.Castro \& Cath. $2 n=56$, seta indicando satélite. (B) K. uniflora (Lindl.) V.P.Castro $2 n=56$. (C) K. unicolor (Rolfe) V.P.Castro \& Cath. $2 n=56$. (D) K. bohnkiana (V.P.Castro \& G.F.Carr) V.P.Castro \& Cath. $2 n=56$, setas indicando satélites. (E) K. longipes (Lindl.) V.P.Castro \& Cath. $2 n=56$. (F) K. croesus (Rchb. f.) V.P.Castro \& Cath. $2 n=56$. Barra 10 micrômetros.

Se mantidos os pequenos gêneros homogêneos o número básico sugerido para Neoruschia seria $x=24$. Este número apenas é relatado para o gênero Macradenia (Tab. 2). Sugerese $x=28$ como número básico para Alatiglossum, Baptistonia, Brasilidium, Carenidium, Coppensia, Kleberiella, Rhinocidium e Ornitophora, pois a maioria dos indivíduos dentro dos gêneros possui $2 n=56$, com algumas variações. Porém, o número básico $x=28$ pode ser referido também para o clado "Gomesa", ou para Gomesa l.s. conforme Chase et al. (2009) e para Oncidium s.s.. Foi observado, também, que a maioria das espécies do gênero Grandiphyllum Docha Neto, que possuem contagens do número cromossômico (Tab. 2), apresentaram $2 n=42$ (Oncidium edwallii, O. harrisonianum e O. pulvinatum), sugerindo que $x=21$ é o número básico para o gênero. Grandiphyllum. Este gênero foi criado a partir da seção Pulvinata Lindl. (Oncidium l.s.), incluindo algumas espécies anteriormente consideradas na seção Ranifera. Fi- logeneticamente é mais próximo do gênero Trichocentrum Poepp. \& Endl. (Chase et al. 2009) o qual também apresenta números cromossômicos mais baixos com variação de $2 n=$ 20, 24, e 28, com provável numero básico $x=14$.

Os resultados obtidos corroboram que muitos gêneros de Oncidiinae possuem o número cromossômico básico $x$ $=28$, como já ressaltado por Félix \& Guerra (2000) e Chase et al. (2005). Observa-se que há dois processos principais na evolução cromossômica no clado "Gomesa" que são a poliploidia e a perda ou acréscimo de pares cromossômicos. Variações semelhantes foram observadas em outros gêneros de Oncidiinae como a perda cromossômica em Macradenia que apresenta espécies com $2 n=48$ e 56, o acréscimo de pares cromossômicos na mesma espécie, como em Miltonia spectabilis com $2 n=56$ e 60, a disploidia em Lockartia com $2 n=14$ e 56, e a poliploidia em Odontoglossum, com $2 n=56$ e 112 (Félix \& Guerra 2000). Estas variações são 

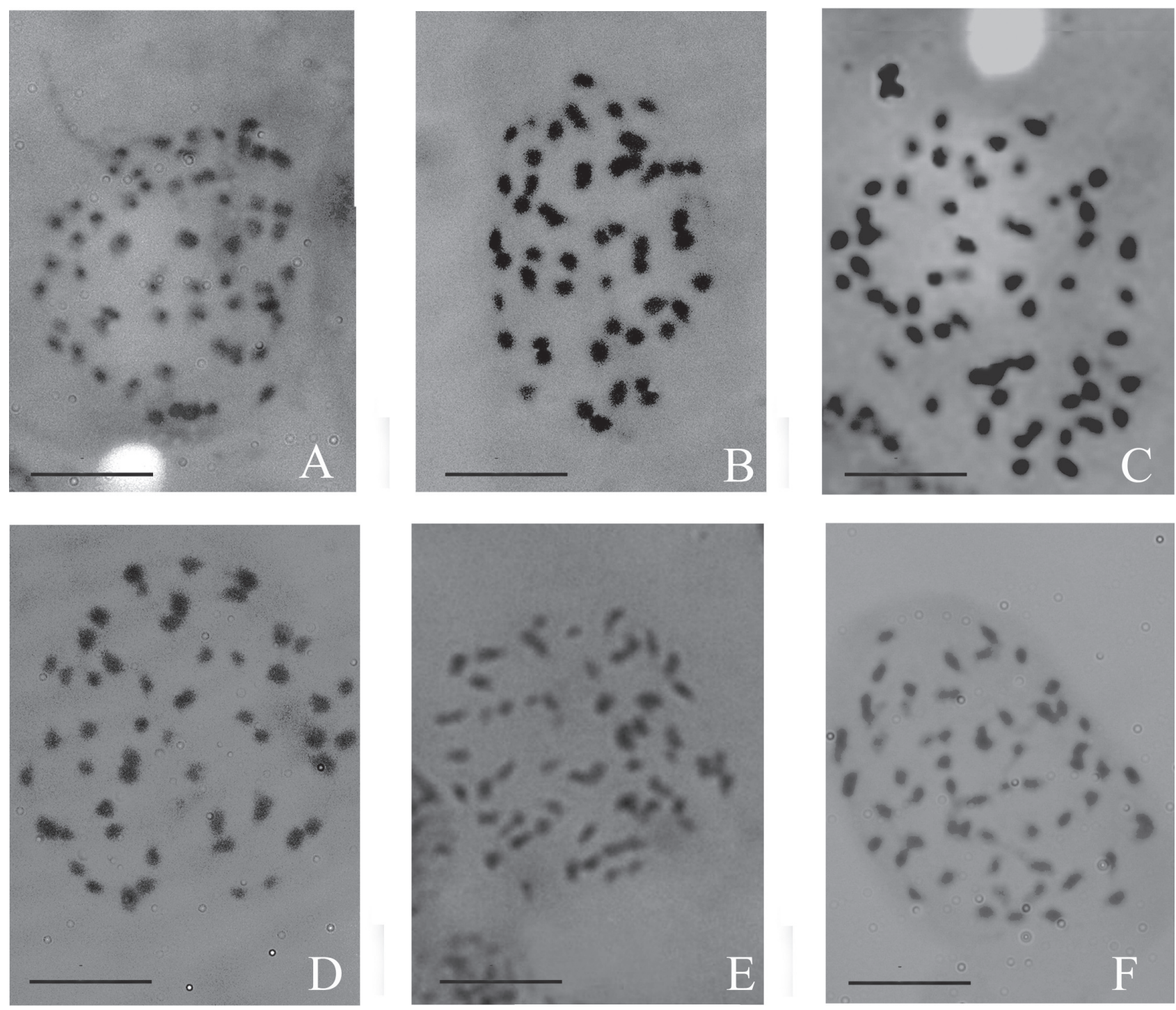

Figura 4. Fotos dos cromossomos das espécies: (A) Alatiglossum ciliatum (Lindl.) Baptista $2 n=56$. (B) Neoruschia cogniauxiana (Schltr.) Cath. \& V.P.Castro $2 n=$ 48. (C) A. barbatum (Lindl.) Baptista $2 n=56$. (D) A. fuscopetalum (Hoehne) Baptista $2 n=52$. (E) A. micropogon (Rchb. f.) Baptista $2 n=56$. (F) Carenidium gracile (Lindl.) Baptista $2 n=54$. Barra 10 micrômetros.

observadas em muitos outros gêneros da subtribo, assim como em gêneros de outras subtribos de Cymbidieae como Christensonella (Maxillariinae) que apresenta espécies com $2 n=36,38$ e 76 (Koehler et al. 2008).

Ainda com relação ao número base para a subtribo Oncidiinae, duas teorias foram propostas, uma sugere que o número básico seja $x=5,6$ e 7 , e séries de poliplóides teriam os números $2 n=28,56,84,112$ e 168 (Félix \& Guerra 2000). Outra teoriza que o número base seria $x=$ 28 e por disploidia teriam sido formados os baixos números, observados em espécies de ciclo curto de vida (Chase et al. 2005). Como é ressaltado que a família tem a origem na Ásia e os gêneros mais antigos no continente asiático relacionados a Oncidiinae como Cymbidium apresentam $2 n$ $=38$ e 40, assim como gêneros mais antigos de distribuição na América como Maxillaria que apresenta $2 n=36,38$ e 40
(Dressler 1993). Assim, seria mais plausível que a subtribo Oncidiinae apresente o número básico mais elevado como $x=28$ do que números baixos como $x=5,6$ ou 7 .

O resultados deste estudo ressaltam a importância do conhecimento do número cromossômico em grupos morfológicos filogeneticamente próximos para a taxonomia e para o entendimento da evolução cromossômica. Os resultados corroboram a posição de que as características vegetativas e mesmos números cromossômicos tendem a sustentar grupos morfológicos filogeneticamente muito próximos, como nos complexos de espécies de Kleberiella e Alatiglossum. Porém, a sua utilização para caracterização de gêneros e espécies deve ser feita com cuidado utilizando outros caracteres em conjunto, respeitando algumas variações do número cromossômico, que podem ser observadas dentro da mesma espécie ou entre espécies relacionadas. 
Tabela 2. Relação de números cromossômicos de alguns gêneros de Oncidiinaelevantados da literatura: Alatiglossum Baptista stricto sensu, Baptistonia Barb. Rodr., Brasilidium Campacci, Carenidium Baptista, Caucaea Schltr., Cohniella Pfitzer, Coppensia Dumort, Erycina Lindl., Gomesa R.Br. stricto sensu, Grandiphyllum Docha Neto, Kleberiella V.P. Castro \& Cath., Lophiaris Raf., Macradenia R.Br., Neoruschia Cath. \& V.P. Castro, Oncidium Sw. Stricto sensu Psychopsis Raf., Phymatochillum Christenson, Rhinocidium Baptista, Rossioglossum (Schltr.) Garay \& C.G. Clein, Tolumnia Raf. E Trichocentrum Poepp. \& Endl. Siglas para os respectivos trabalhos: B60 = Blumenschein (1960); CK75 = Charanasri \& Kamemoto (1975); CK73 = Charanasriet al. (1973); S69 = Sinotô (1969); TK84 = Tanaka \& Kamemoto (1984); FG00 = Félix \& Guerra (2000); DV09 = Davinãet al. (2009); M73 = Moore (1973); M74 = Moore (1974); M77 = Moore (1977); NT $=$ No presente trabalho.

\begin{tabular}{|c|c|c|c|}
\hline Táxon & & Número diplóide (número haplóide) & Referência \\
\hline \multirow[t]{4}{*}{ Alatiglossum } & barbatum & $(28)$ & B57,FG00, NT \\
\hline & ciliatum & 56 & NT \\
\hline & micropogon & 56 & TK84,NT \\
\hline & fuscopetalum & 52 & NT \\
\hline \multirow[t]{4}{*}{ Baptistonia } & echinata & 56 & TK84 \\
\hline & pubes & 84 & DV09 \\
\hline & riograndense & 56 & DV09 \\
\hline & sarcodes & 56 & TK84 \\
\hline \multirow[t]{5}{*}{ Brasilidium } & crispum & 56 & TK84 \\
\hline & curtum & 52 & TK84 \\
\hline & gravesianum & 56 & FG00 \\
\hline & marshalianum & 56 & TK84 \\
\hline & praetextum & (28) & TK84, FG00 \\
\hline Carenidium & gracile & 54 & NT \\
\hline Caucaea & cucullata & 54 & TK84 \\
\hline \multirow[t]{3}{*}{ Cohniella } & cebolleta & $36,72(18)$ & TK84,FG00 \\
\hline & jonesiana & 30 & TK84,FG00 \\
\hline & nuda & 36 & TK84,FG00 \\
\hline \multirow[t]{6}{*}{ Coppensia } & bifolia & 108 & DV09 \\
\hline & blanchetii & ca. 112 & FG00 \\
\hline & flexuosa & 56 & TK84,FG00,MB10 \\
\hline & aff. flexuosa & ca. 168 & FG00 \\
\hline & varicosa & $56,112 / 168(28,56)$ & S69,TK84, FG00 \\
\hline & warmingii & 140 & TK84, FG00 \\
\hline \multirow[t]{2}{*}{ Erycina } & glossomystax & 14 & TK84 \\
\hline & pusilla & 10,14 & S69,TK84, FG00 \\
\hline \multirow[t]{2}{*}{ Gomesa } & crispa & 56 & FG00 \\
\hline & recurva & 56 & FG00 \\
\hline \multirow[t]{5}{*}{ Grandiphyllum } & harrisonianum & 42 & FG00 \\
\hline & divaricatum & $42 / 56$ & CK73/DV09 \\
\hline & edwallii & 42 & DV09 \\
\hline & robustissimum & 44 & TK84,FG00 \\
\hline & pulvinatum & 42 & M77,FG00 \\
\hline \multirow[t]{6}{*}{ Kleberiella } & bohnkiana & (28)56 & B57,DV09,NT \\
\hline & croesus & 56 & NT \\
\hline & longipes & 56 & NT \\
\hline & regentii & 56 & NT \\
\hline & uniflora & 56 & NT \\
\hline & unicolor & 56 & NT \\
\hline \multirow[t]{14}{*}{ Lophiaris } & carthaginense & 30 & TK84 \\
\hline & bicallosa & $28(14)$ & CK75 \\
\hline & cavendishiana & (14) & B57,FG00 \\
\hline & haematochila & 28 & FG00 \\
\hline & lanceana & 26,28 & FG00 \\
\hline & lurida & $28,31,32$ & S69,TK84, \\
\hline & microchila & $36,37,38$ & CK73, \\
\hline & morenoi & 30 & D97 \\
\hline & nana & 26 & TK84, \\
\hline & oestlundiana & 28 & TK84, \\
\hline & pumilla & 30 & TK84, D97,FG00 \\
\hline & stipitata & 36 & TK84, FG00,MB10 \\
\hline & splendida & 36 & M77 \\
\hline & straminea & 28,30 & S69,TK84, \\
\hline Macradenia & brassavolae & 48 & TK84 \\
\hline
\end{tabular}


Tabela 2. Continuação

\begin{tabular}{|c|c|c|c|}
\hline Táxon & & Número diplóide (número haplóide) & Referência \\
\hline & paraensis & (28) & B57 \\
\hline Neoruschia & cogniauxiana & 48 & NT \\
\hline \multirow[t]{45}{*}{ Oncidium } & altissimum & 56 & S69 \\
\hline & ansiferum & 56 & S69 \\
\hline & anthrocrene & 56 & S69 \\
\hline & aurosum & 54 & TK84,FG00 \\
\hline & brachyandrum & 56 & S69 \\
\hline & baueri & 56 & FG00 \\
\hline & cheirophorum & 56 & TK84, \\
\hline & cheirophorum & 56 & TK84 \\
\hline & ensatum & 56 & FG00 \\
\hline & excavatum & 56 & FG00 \\
\hline & floridanum & 56 & FG00 \\
\hline & fimbriatum & 56 & DV09 \\
\hline & globuliferum & 56 & CK75 \\
\hline & hastatum & 56 & TK84,FG00 \\
\hline & hieroglyphicum & 56 & TK84,FG00 \\
\hline & hyphaemacticum & 56 & FG00 \\
\hline & incurvum & 56 & M73,FG00 \\
\hline & inouei & 56 & FG00 \\
\hline & isthmi & 56 & CK75 \\
\hline & nebulosum & 56 & TK84,FG00 \\
\hline & nigratum & 56 & TK84,FG00 \\
\hline & obryzatoides & 56 & TK84,FG00 \\
\hline & obryzatum & 56 & TK84,FG00 \\
\hline & onustum & 56 & TK84,FG00 \\
\hline & ornithorrhynchum & $56(28)$ & TK84,FG00 \\
\hline & panamense & 56 & TK84,FG00 \\
\hline & parviflorum & 56 & TK84,FG00 \\
\hline & pentadactylon & $40 / 42$ & TK84,FG00 \\
\hline & polyandenium & 56 & M77,FG00 \\
\hline & leuchochilum & 56 & TK84,FG00 \\
\hline & lammeligerum & 55,57 & TK84,FG00 \\
\hline & powelli & 56 & S69 \\
\hline & sphacelatum & 56 & S69 \\
\hline & trilobum & 56 & GJ94, FG00 \\
\hline & triquetrum & 56 & CK73 \\
\hline & trilobum & 56 & CK73 \\
\hline & volvox & (28) & TK84, FG00 \\
\hline & stenotis & 56 & S69,FG00 \\
\hline & tetraskelidon & (28) & TK84, FG00 \\
\hline & tigrinum & 56 & FG00 \\
\hline & maculatum & 56 & M73,FG00 \\
\hline & longifolium & (28) & TK84,FG00 \\
\hline & loxense & 56 & TK84,FG00 \\
\hline & phalaenopsis & 56 & TK84,FG00 \\
\hline & wentworthianum & 56 & FG00 \\
\hline \multirow[t]{2}{*}{ Psychopsis } & krameriana & 38 & TK84,FG00 \\
\hline & papilio & 38 & FG00 \\
\hline Phymatochilum & brasiliesnsis & 56 & M73 \\
\hline \multirow{3}{*}{ Rhinocidium } & longicornum & $56 / 42$ & DD99/DV09 \\
\hline & loefgrenii & $56(28)$ & FG00 \\
\hline & paranaense & 56 & FG00 \\
\hline Rossioglossum & ampliatum & 44 & S69,CK75 \\
\hline \multirow[t]{2}{*}{ Tolumnia } & desertora & 40 & M77 \\
\hline & intermedia & 40 & CK75 \\
\hline \multirow[t]{3}{*}{ Trichocentrum } & albo-purpureum & 24,28 & TK84 \\
\hline & capistratum & 28 & TK84 \\
\hline & cornucopiae & 20 & FG00 \\
\hline
\end{tabular}




\section{Agradecimentos}

Agradecemos à Vitorino P. Castro Neto pelo fornecimento de parte dos materiais para as análises e pela sua disponibilidade em colaborar, ao Núcleo do Orquidário (Orquidário Frederico Carlos Hoehne), Instituto de Botânica de São Paulo pela cessão de uso das plantas, aos curadores Maria Candida H. Mamede (SP) e João Aurélio Pastore (SPSF) pela permissão de depósito dos materiais analisados; ao CNPQ (proc. 005353/2009) pela bolsa de mestrado concedida ao primeiro autor, junto ao programa de Pós-Graduação em Biodiversidade Vegetal e Meio Ambiente do Instituto de Botânica de São Paulo e aos dois assessores deste artigo, pelos valiosos comentários.

\section{Referência Bibliográficas}

Barros, F. \& Rodrigues. V.T. 2010. Novas combinações para membros brasileiros da subtribo Oncidiinae (Orchidaceae, Epidendroideae, Cymbidieae). Boletim CAOB 77-78: 3-15.

Blumenschein, A. 1960. Número cromossômico de algumas espécies de orquídeas. Publicações Cientificas da Universidade de São Paulo 1:45-50.

Castro Neto, V.P. \& Catharino, E.L.M. 2006. Kleberiella et Neoruschia (Orchidaceae, Onciidinae) deux nouveaux genre extraits du genre Alatiglossum. Richardiana 6(3): 148-160.

Catharino, E.L.M. \& Campacci, M.A. 2009. Propostas para novas combinações nomenclaturais. Boletim CAOB 70: 95-97.

Charanasari, U. \& Kamemoto, H. 1975. Additional chromosome numbers in Oncidium and allied genera. American Orchid Society Bulletin 44: 686-691.

Charanasari, U.; Kamemoto, H. \& Takashita, M. 1973. Chromosome numbers in the genus Oncidium and some allied genera. American Orchid Society Bulletin 42: 518-524.

Chase, M.W. 1986. A reppraisal of the oncidioid orchids. Sytematic Botany 11(3): 477-491.

Chase, M.W.; Cameron, K.M.; Barrett, R.S.; Freudenstein, J.V. 2003. DNA data and Orchidaceae systematic: a new phylogeny classification. Pp. 69-89. In: Dixon, K.W.; Kell, S.P.; Barrett, R.L.; Cribb, P.J. (Eds.). Orchid Conservation. Sabah, Natural History Publications, Kota Kinabalau.

Chase, M.W.; Hanson, L; Albert, V.A; Witthen, W.M. \& Willians, N.H. 2005. Life history evolution e genome size in subtribe Oncidiinae (Orchidaceae). Annals of Botany 95: 91-199.

Chase, M.W.; Williams, N.H.; Faria, A.D.; Neubig, K.M.; Amaral, M.C.E.; Whitten, M.W. 2009. Floral convergence in Oncidiinae (Cymbidieae; Orchidaceae): an expanded concept of Gomesa and a new genus Nohawilliamsia. Annals of Botany 104(3): 397.

Chase, M.W. \& Palmer, J.D. 1992. Floral morphology and chromosome number in subtribe Oncidiinae (Orchidaceae): evolutionary insights from a phylogenetic analysis of chloroplast DNA restriction site variation. Pp. 324-339. In: Soltis, D.E.; Soltis, P.S.; Doyle, J.J. (Eds.) Molecular Systematic of Plants. New York, Chapman and Hall.

Corrêa, A.M. 2003. Estudos cromossômicos em espécies de Rubiaceae Juss. de Cerrado. Dissertação de Mestrado. Campinas, Universidade Estadual de Campinas.

Cozzollino, S.; D’Emerico, S.; Widmer, A. 2004. Evidence for reproductive isolate selection in Mediterranean orchids: kariotype differences compensate for the lack of pollinator specificity. The Royal Society, Biology Letters 271: 259-262.

Davinã, J.R.; Grabiele, M.; Cerutti, J.C.; Hojsgaard, D.H.; Almada, R.D.; Insaurralde, I.S.; Honfi, A.I. 2009. Chromosome studies in Orchidaceae from Argentina. Genetics and Molecular Biology 32(4): 811-821.

Dematteis, M. 1997. The kariotypes of Oncidium morenoi and O. pumilum (Orchidaceae). Selbyana 18(2): 186-187.
Docha Neto, A.; Batista, D.H. \& Campacci, M.A. 2006. Novos gêneros (baseados em Oncidium). Coletânea de Orquídeas Brasileiras 3: 67-94. São Paulo, Editora Brasil Orquídeas.

Dressler, R.L. 1993. Phylogeny and classification of the orchid family. Portland, Dioscorides Press.

Faria, A. 2004. Sistemática filogenética e delimitação dos gêneros da subtribo Oncidiinae (Orchidaceae) endêmicos do Brasil: Baptistonia, Gomesa, Ornithophora, Rodrigueziella, Rodrigueziopsis e Oncidium pro parte. Tese de Doutorado. Campinas, Universidade Estadual de Campinas.

Félix, L.P. \& Guerra, M. 2000. Cytogenetics and cytotaxonomy of some brazilian species of Cymbidioid orchids. Genetics and Molecular Biology 23 (4): 957-978.

Francesca, B. \& Aquaro, G. 2008. Contribution to the cytotaxonomical knowledge of four species of Serapias L. (Orchidaceae). Caryologia 61(3): 294-299.

Goldblatt, P. (Ed.) 1984. Index to Plant Chromosome Numbers 19791981. Saint Louis, Missouri Botanical Garden.

Goldblatt, P. (Ed.) 1985. Index to Plant Chromosome Numbers 1982 1983. Saint Louis, Missouri Botanical Garden.

Goldblatt, P. \& Johnson, D.E. (Eds.) 1988. Index to Plant Chromosome Numbers 1984-1985. Saint Louis, Missouri Botanical Garden.

Goldblatt, P. \& Johnson, D.E. (Eds.) 1990. Index to Plant Chromosome Numbers 1986-1987. Saint Louis, Missouri Botanical Garden.

Goldblatt, P. \& Johnson, D.E. (Eds.) 1991. Index to Plant Chromosome Numbers 1988-1989. Saint Louis, Missouri Botanical Garden.

Goldblatt, P. \& Johnson, D.E. (Eds.) 1994. Index to Plant Chromosome Numbers 1990-1991. Saint Louis, Missouri Botanical Garden.

Goldblatt, P. \& Johnson, D.E. (Eds.) 1996. Index to Plant Chromosome Numbers 1992-1993. Saint Louis, Missouri Botanical Garden.

Guerra, M. 1983. O uso de Giemsa na citogenética vegetal. Comparação entre a coloração simples e o bandeamento. Ciência e Cultura 35: 190-193.

Koehler, S.; Cabral, J.S.; Whitten, W.M.; Williams, N.H.; Singer, R. B.; Neubig, K.M.; Guerra, M.; Souza, A. P.; Amaral, M.C.E. 2008. Molecular phylogeny of Neotropical genus Christensonella (Orchidaceae, Maxillarinae): species delimitation and insights into chromosome evolution. Annals of Botany 102: 491-507.

Moore, R.J. (Ed.) 1973. Index to plant chromosome number 1967-1971. Regnum Vegetabile 90: 1-539.

Moore, R.J. (Ed.) 1974. Index to plant chromosome number 1972. Regnum Vegetabile 91: 1-108

Moore, R.J. (Ed.) 1977. Index to plant chromosome number 1973-1974. Regnum Vegetabile 96:1-157

Penha, T.L.L. 2011. Taxonomia e conservação de Kleberiella V.P. Castro \& Cath. (Orchidaceae, Oncidiinae). Dissertação de Mestrado. São Paulo, Instituto de Botânica de São Paulo.

Penha, T.L.L. \& Catharino, E.L.M. 2010. Binotia messmeriana e seu posicionamento taxonômico. Boletim CAOB 77-78: 16-19.

Pridgeon, A.M., Cribb, P.J., Chase, M.W. \& Rasmussen, F.N. 2009. Genera Orchidacearum 5: Epidendroideae (part two). New York, University Press.

Sinotô, Y. 1969. Chromosomes in Oncidium and allied genera, I. Genus Oncidium. Kromossomo 76: 2459-2473.

Soltis, P.S. \& Soltis, D.E. 2009. The role of hybridization in plant speciation. Annual Review of Plant Biology 60: 561.

Stace, C.A. 1989. Plant taxonomy and biosystematics. 2nd ed. New York, University Press, Cambridge.

Stebbins, G. L. 1971. Chromosomal evolution in higher plants. London, Edward Arnold.

Tanaka, R. \& Kamemoto, H. 1984. Chromosomes in orchids: counting and number. Pp. 234-283. In: Arditti, J. (Ed.). Orchid Biology: Reviews and Perspectives III. Ithaca, Cornell University Press.

Teuscher, H. 1968. Oncidium uniflorum and Oncidium longipes. American Orchid Society Bulletin 37(1-6): 51-54.

Williams, N.H., Chase, M.W.; Fulcher, T. \& Whitten, W.M. 2001. Molecular systematic of the Oncidiinae based on evidence from four DNA sequence regions: expanded circumscription of Cyrtochilum, Erycina, Otoglossom, and Trichocentrum and a new genus (Orchidaceae). Lindleyana 16(2): 113-139. 